\title{
Behaviour Research and Therapy-Predictors of Obsessive Compulsive Disorder: A Preliminary Investigation
}

\author{
Kristina Radzeviciute* \\ Department of Psychology, Kingston University London, UK
}

Submission: April 11, 2017; Published: April 28, 2017

*Corresponding author: Kristina Radzeviciute, Kingston University London, Department of Psychology, Penrhyn Road, Surrey, KT1 2EE, UK, Email: kristina_rad@hotmail.co.uk

\begin{abstract}
This study investigated predictor factors of obsessive compulsive disorder in university student population.

Method: Ninety-six participants completed various tests that measured demographic factors (age, ethnicity, relationship status, educational level and employment status), innate factors (history of substance use and life events over the last year) and psychiatric comorbidity (mood and anxiety disorders).
\end{abstract}

Results: Depression was the strongest predictor of obsessive compulsive disorder (OCD) General anxiety and life events stress was also a positive predictor of $\mathrm{OCD}$.

Conclusion: Depression is the strongest predictor of OCD. General anxiety and life events stress acts as positive predictors of OCD.

Keywords: Obsessive Compulsive Traits; demographics (age, ethnicity, relationship status, educational level, employment status); innate factors (substance use, stressful life events); psychiatric comorbidity (depression, anxiety disorders); preliminary study; quantitative study; students

Abbreviations: OCD: Obsessive Compulsive Disorder; DSM-5: Diagnostic and Statistical Manual of Mental Disorders; MZ: Mono-Zygotic; DZ: Di-Zygotic; GABBR-1: GABA type B Receptor 1; PHQ-9: Patient Health Questionnaire; GAD-7: General Anxiety Disorder Questionnaire; OCI: Obsessive Compulsive Inventory; SRRS: Social Readjustment Rating Scale; SPSS: Statistical Package for the Social Sciences

\section{Introduction}

Obsessive Compulsive Disorder (OCD) fall under the Obsessive-compulsive and Related Disorders spectrum of the Diagnostic and Statistical Manual of Mental Disorders (DSM-5). The DSM-5 defines OCD as a mental health disorder characterized by an individual showing the presence of obsessions and /or compulsions. Obsessions are thoughts, urges or images that persist over time and are distressful, intrusive and unwanted. On the other hand, compulsions are physical and/or mental behaviours or mental acts that are repeated as a response to an obsession/s for the purpose of reducing anxiety. There are many subtypes of OCD including: contamination (physical and mental), hoarding, intrusive thoughts, symmetry, orderliness, rumination and checking. It is estimated that OCD currently affects $0.3 \%$ to $3.1 \%$ of the population. OCD typically develops during childhood and early adulthood.
Research into the causal factors of OCD have identified factors including genetics, neurochemical functioning and brain functioning. I will discuss each of these causal factors in greater depth. First of all, research has shown evidence to suggest that genetics play a major role in OCD. Twin and family studies report evidence which shows that OCD tends to run in families. Gottesman [1] found a concordance rate for OCD of monozygotic (MZ) twins to be $80-87 \%$ in comparison to $47-50 \%$ in dizygotic (DZ) twins. Further research by Paul, Alsobrook, Goodman, Rasmussen and Leckman [2] reports that first degree relatives of individuals diagnosed with OCD are at a 10\% higher risk of also developing OCD when compared to controls.

Furthermore, although limited, numerous studies suggest that specific genes are responsible for OCD. Willour et al. [3] found that one of these is the genome on region 9p24 at marker 
D9S288. Additionally, Delorme et al. [4] found evidence to suggest gene association between SLC6A4 V25 and OCD as they found a higher occurrence of this gene in participants diagnosed with OCD in comparison to control subjects. Findings reported by Ozaki et al. [5] also suggest that participants that presented with the most severity of their OCD symptoms had an I425V SERT gene mutation and were homozygous for 5'-UTR SERT gene which is responsible for transcriptional efficacy. Additionally, Arnold et al. [6] looked at OCD probands and their families and found evidence that over transmission at the A-7265G polymorphism is evidence in individuals diagnosed with OCD, suggesting an association between GABA type B receptor 1 (GABBR1) gene and OCD. Some studies have also found an associated between glutamate (NMDA) subunit receptor gene (GRIN2B) and OCD.

Moving onto neurochemical functioning in OCD, research has shown that the serotonergic system may play a role in OCD. In particular, Marazziti, Hollander, Lensi, Ravagli and Cassano [7] reported a dysfunction of serotonin on the peripheral markers for the 5-HT in the form of basal hyper activation present either presynaptically or postsynaptically, involved in neurotransmission and self-regulation. Mundo, Bereggi, Pirola and Bellodi [8] reported that patients that received clomipramine administration as treatment for OCD have reported improvement in their symptoms. Additionally, David, Charney, Coyle and Nemeroff [9] studies looking at the role dopamine plays in OCD, has found that dopamine antagonists have been found to be effective in treating OCD.

Another causal factor in OCD is brain functioning. Saxena and Rauch [10] found evidence of significant abnormities and dysfunction in the form of elevated brain activity in the orbitofrontal cortex, basal ganglia and stratum in individual diagnosed with OCD. Additionally, Simon, Adler, Kaufman and Kathman [11] found evidence to suggest an increased activity in the fronto-striato-limbic circuits, including the amygdala in individuals diagnosed with OCD. Heyman et al. [12] reported evidence of amygdala hyper activation, possible accounting for the increased fear response to specific stimuli (obsessions) observed in individuals diagnosed with OCD.

Having said this, this study looked into the predictor factors of OCD. There has been limited research into the predictor factors of OCD. However, a meta-analysis identified the following factors as predictors of OCD: demographics (age, ethnicity, relationship status, educational level and employment status), innate factors (participant and familial psychiatric history and background of OCD), environmental factors (substance abuse and life events) and psychiatric comorbidity (major depressive disorder and anxiety disorders). I will now discuss each of these predictor factors in greater depth. First of all, age has been reported to be a potential predictor factor of OCD. Willour et al. [3] reported evidence suggesting OCD becomes more common with increase in age. Additionally, Heyman et al. [12] found that the age of onset of OCD usually begins in childhood and early adulthood. However, there is a lot of dispute about the actual age of onset.
Having said this, ethnicity has been shown to play a major role in OCD. Earlier studies have reported that individuals from minority ethnic groups have a greater risk of enveloping OCD. Heyman et al. [12] found a greater proportion of OCD in children from ethnic minorities. Similarly, Williams, Turkheimer, Schmidt and Oltmanns [13] found that OCD treatment seeking is significantly greater from minority ethnic groups. However, more research needs to be conducted in order to investigate this further and to identify explanations to account for the greater risk minority ethnic groups possesses when it comes to developing OCD. One major criticism of research into the impact of race and ethnicity on the risk of developing OCD concerns the fact that many studies have predominantly focused on individuals from a white ethnic group.

Another important predictor factor is the relationship status of the individual diagnosed with OCD. A study by Cilli et al. [14] on 3,012 adults diagnosed with OCD found that individuals diagnosed with OCD were 4.2 times more likely to be divorced, separated, or widowed when compared to controls. However, some studies including a study by Crino, Slade and Andrews [15] have found no difference in the marital status of individuals diagnosed with OCD when compared to controls. Furthermore, Fontenelle, Mendlowicz, Marques ad Versiani [16] found that individuals diagnosed with OCD tend to have higher educational achievements, therefore suggesting that educational level may play a predictive role in OCD. Similarly, Dekonda, Wyss ad Angst [17] found similar results. However, Crino, Slade and Andrews [15] were unable to replicate this finding and there this presumption can be questioned.

To add, research has shown that employment status may also play a predictive role in OCD. Some research suggests major differences between individuals diagnosed with OCD and healthy controls when it comes to employment statuses. Fontenelle, Mendlowicz, Marques and Versiani [16] reported high unemployment rates in those diagnosed with OCD when compared to controls. On the contrary, other research has found no significant difference in employment between participants diagnosed with OCD and controls. Therefore, more research needs to be conducted for conclusions to be made. Moving into, environmental factors, substance abuse and life events stress have both been shown to have a predictive effect on the likelihood of someone developing OCD. When it comes to substance abuse, research shows that substance abuse could act as a predictor of OCD. A study by Douglass, Moffitt, Dar, McGee and Silva [18] looked at a birth cohort of 18 year olds; found that $24 \%$ of individuals diagnosed with OCD met the criteria for alcohol dependence. Further research by Moyla, Jacka, Pasco and Berk [19] reported that individuals who use tobacco and are dependent are more likely to be diagnosed with OCD as well as other anxiety disorders.

Furthermore, another environmental factor that has shown to predict OCD is the presence of stressful life events. ValleniBasile et al. [20] found that individuals who have experienced 
the most undesirable or stressful life events were at higher risk of developing OCD. Additionally, in the general population, Maues, Mylle, Delmeire and Altamura [21] found a significant correlation has also been found between life events, trauma and the development of OCD. Lastly, research shows evidence to suggest that psychiatric comorbidity (depressive and anxiety disorders) may act as predictors of OCD. Kessler et al. [22] has shown that OCD has a strong link with mood disorders such as major depressive disorder and anxiety disorders such as generalized anxiety disorder. They conducted their research on 9,282 English speaking participants. They looked at the lifetime prevalence estimates of OCD in relation to other disorders. They found estimate prevalence between OCD and mood disorders to be $20.8 \%$ and $28.8 \%$ between OCD and anxiety disorders.

\section{Methods}

\section{Participants}

A sample of 96 (75 females, 21 males) participants were recruited from Kingston Univerity London, Penrhyn Road Campus, through the SONA voluntary system. The inclusion criteria required them to be a student at Kingston University London and be at least 18 years of age. The age ranged from 18 to 59 years of age $(M=24.06, S D=7.7)$. The study took place in a dedicated psychology laboratory room at Kingston University London. The sample comprised of 47 Caucasians, 22 African/Caribbean's, 16 Asians, 7 Mixed and 4 others. In terms of relationship status, 48 were single, 39 were in a relationship, 8 were married and 1 was separated. The educational attainment of the sample were as follows: 2 participants reported to have GCSE's, 45 reported to have A levels or BTEC, 5 had MCQs or diploma, 35 had an Undergraduate degree and 9 had a postgraduate degree as their highest level of education.

Additionally, 52 participants were employed with student status and 44 were not working while being a university student. The study required all participants to state their use of substances. It was reported that 4 participants engaged in smoking behaviour, 53 reported alcohol use behaviour, 17 reported both smoking and alcohol use behaviour and 22 participants reported to using no substances. Lastly, the psychiatric history of the participants and their families showed that 8 participants reported to have a diagnosis of a psychiatric condition, 28 reported to have a family member diagnosed with a psychiatric condition, 11 reported to have a diagnosis of a psychiatric condition as well as having someone in their family diagnosed with one and lastly, 49 reported to having no diagnosis of a psychiatric condition themselves and neither their family. The study was approved by the internal departmental Research Ethics Committee.

\section{Assessment}

A demographics questionnaire was used to gather data on the age, gender, ethnicity, employment status, relationship status and education level of the participants. A substance use questionnaire was used to measure whether the participants consumed alcohol and/or participated in smoking. Additionally, the Patient Health Questionnaire (PHQ-9) developed by Kroenke and Spitzer [23] which is a 9 item self-reported questionnaire was used to measure and assess mood and depression. The participants were provided with a total of 9 statements and asked to write a response to indicate how often they have been bothered by the stated problem over the last two weeks. For example, the participants were asked how often over the last two weeks they have experienced 'Little interest or pleasure in doing things' and experienced 'feeling down, depressed, or hopeless'.

The General Anxiety Disorder Questionnaire (GAD-7) developed by Spitzer, Kroenke, Williams, and Löwe [24] which is a 7-item self-reported questionnaire which was used to measure and assess severity of general anxiety disorder. The participants were provided with 7 statements and were asked to write a response to indicate how often they have been bothered by an indicated problem over the last two weeks. For example, the participants were asked how often over the last two weeks they have been 'Feeling nervous, anxious or on edge' and experienced 'Not being able to stop or control worrying'. The total score indicated severity of generalized anxiety disorder (GAD).

Furthermore, the Obsessive Compulsive Inventory (OCI) questionnaire developed by Foa, Kozak, Salkovskis, Coles, \& Amir [25] which is a 42-item self-reported scale was used to measure the presence of obsessive compulsive traits. The participants were provided with statements and asked to write a response to indicate how much a specified experience has distressed or bothered during the past month. For example, the participants were given the following statement 'Unpleasant thoughts come into my mind against my will and I cannot get rid of them' and I wash and clean obsessively' and were asked to indicate how often this bothered them. The statements varied and measured different subtypes of OCD including washing, checking, doubting, ordering, obsessing, hoarding, and mental neutralising. The total score indicated severity of OCD and the traits associated.

Another scale was the Holmes-Rahe Social Readjustment Rating Scale (SRRS). The SRRS was developed by Holme and Rahe [26] and was used to measure the amount of stressful life events that a participant has experienced which indicated the extent of risk the participant has in experiencing stress-related illness. Lastly, a family background and history questionnaire was used to assess history of possible psychiatric disorders in the family, with the option to not disclose this information. The psychiatric conditions that were assessed included bipolar and related disorders, depressive disorders, anxiety disorders, schizophrenia spectrum and other psychotic disorders, trauma and stressor related disorders, feeding and eating disorders, substance related and addictive disorders, personality disorders and other unspecified disorders. The participants could tick as many psychiatric disorders as they wished if relevant. 


\section{Procedure}

A total of 96 participants were recruited through the SONA voluntary system at Kingston University London. The participants viewed the study on the SONA system and signed up for an individual timeslot. Prior to the timeslot, the participants were sent an e-mail, reminding them of their timeslot and the laboratory room number. On the day of the timeslot, the participants were thanked for coming and asked to take a seat. Following this, an information sheet was provided and they were given the opportunity to ask any questions. If participants agreed to take part in the study, they were provided with a consent form to sign to collect informed consent.

Following this, the participants received a 'questionnaire pack' which consisted of the questionnaires in the following order: demographics questionnaire, substance use questionnaire, patient health questionnaire, general anxiety disorder questionnaire, obsessive compulsive inventory (OCI) questionnaire, Holmes-Rahe Social Readjustment Rating Scale (SRRS) questionnaire and family background and history questionnaire. The participants were asked to complete the questionnaire pack and were informed that that they can withdraw from the study at any point if any form of distress is experienced. Once the questionnaire pack was completed, the participants were given a debrief sheet and given the chance to ask any questions regarding their participation, the study and previous research into the predictor factors of OCD. Lastly, the participants were thanked for their participation and were administered SONA credits.

\section{Statistical analysis}

Version 22 of the Statistical Package for the Social Sciences (SPSS) was used to report frequencies and descriptives. Additionally, multiple regressions was used to investigate into the predictor factors of OCD, to identify the dependent variable with the greatest variance in predicting OCD. Having said this, in order to investigate the association between the independent variables and the dependent a Pearson's $r$ correlational coefficient analysis was conducted.

\section{Results}

The study used a cross-sectional design. OCD was the independent variable and demographic factors demographics (age, ethnicity, relationship status, educational level and employment status), innate factors (participant and familial background and history of OCD), environmental factors (substance abuse and life events) and psychiatric comorbidity (mood and anxiety disorders) were the independent variables.

\section{Descriptive statistics}

Table 1 indicates an age range of 18 to 59. Using score guide for each of the study variables revealed that $34.6 \%$ of the participants reported experiencing mild depression and $26.9 \%$ experienced moderate to severe depression. When it comes to anxiety, $28.9 \%$ experienced mild anxiety and $20.2 \%$ experienced moderate to severe anxiety. Furthermore, when looking at the OCD traits although the mean OCD score is below the required $42+$ score to be considered as diagnosable OCD, the score indicated a medium variance of OCD traits amongst the university student population. Additionally, $16.3 \%$ of the participants met the criteria for OCD and $2.9 \%$ had a diagnosis of OCD. Lastly, $48 \%$ of the participants were at a moderate risk of illness due to stress and $35.9 \%$ were at a significant increase risk of illness.

Table 1: Shows a summary of means and standard deviations for study variables age, depression, general anxiety, obsessive compulsive disorder and life events.

\begin{tabular}{|c|c|}
\hline Variable & Mean, standard deviation \\
\hline Age & $24.06 / 7.72$ \\
\hline PHQ-9 & 7.33 .4 .66 \\
\hline GAD-7 & $6.16 / 4.78$ \\
\hline OCI & $28.86 / 21.61$ \\
\hline SRRS & $248.41 / 123.52$ \\
\hline
\end{tabular}

*Note: PHQ-9: Patient Health Questionnaire; GAD-7: Generalized Anxiety Disorder; OCl: Obsessive Compulsive Disorder; SRRS: Holmes and Rahe Life events stress scale.

\section{Multiple regression analysis}

Predictive variance between all the independent variables and OCD: Stepwise method for multiple regression revealed that depression was the best predictor of the development of obsessive-compulsive traits and OCD as it explained $24 \%$ of the variance $F(1,94)=.29 .65, p<.01, R 2=.24$

Relationship between depression and OCD: A Pearson correlation coefficient revealed a significant positive correlation between obsessive-compulsive traits, OCD and depression, $\mathrm{r}(96)$ $=.49, \mathrm{p}=.01$.

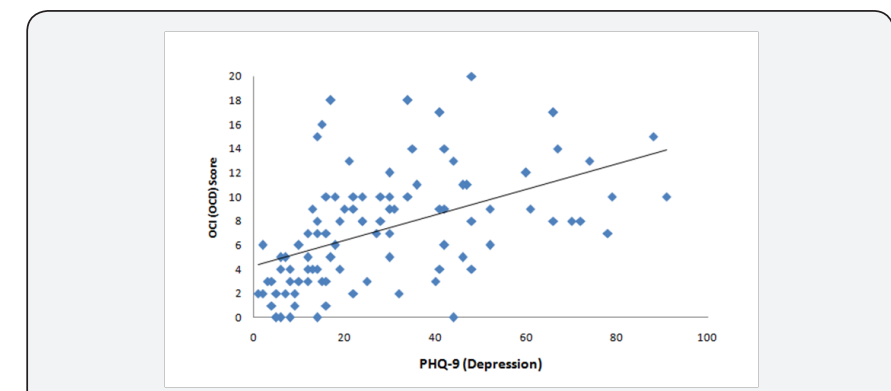

Figure 1: Shows a scatter plot showing the association between depression and obsessive-compulsive traits and OCD.

Figure 1 depicts a significant medium positive correlation between depression and obsessive-compulsive traits and OCD, $\mathrm{r}(96)=.49, \mathrm{p}<.01$. This indicates that the score which indicates that the presence of depression, increases an individual's likelihood of also developing obsessive-compulsive traits and OCD. 


\section{Correlations between all variables}

Correlational bivariate analysis revealed no significant correlation between the independent variables (age, ethnicity, relationship status, educational level, employment status, substance use, and family background and history of OCD) and the independent variable obsessive-compulsive traits and OCD. However, as indicated by Table 2, significant positive correlations was found between depression and obsessive-compulsive traits ( $\mathrm{r}=.490, \mathrm{p}<.01)$, anxiety and obsessive-compulsive traits ( $\mathrm{r}=.386$, $\mathrm{p}<0.1$ ) and stressful life events and obsessive-compulsive traits $(\mathrm{r}=.205, \mathrm{p}<.05)$.

Table 2: Shows the summary of all significant correlations.

\begin{tabular}{|c|c|c|c|}
\hline Measure & Depression & Anxiety & $\begin{array}{c}\text { Stressful Life } \\
\text { events }\end{array}$ \\
\hline OCI (OCD) & $.490^{* *}$ & $.386^{* *}$ & $.205^{*}$ \\
\hline
\end{tabular}

*Note: The table shows Pearson correlation coefficients ( $r$ ) for the independent variables found to be significant with $\mathrm{OCl}(\mathrm{OCD}) .{ }^{*} \mathrm{p}<.05$, ${ }^{* *} \mathrm{p}<.01$

\section{Discussion}

The study found that depression was the strongest predictor of the development of OCD and contributed to $24 \%$ of the variance of the obsessive compulsive trait severity as judged by the total score on the obsessive compulsive inventory. Additionally, the study found a medium positive correlation between anxiety, life events stress and OCD. Looking at previous research, there is limited evidence to suggest that depression may act as one of the potential strongest predictors of the development of OCD. Fullana et al. [27] conducted a cross-sectional survey which assessed the obsessive-compulsive symptom dimensions through the use of the Composite International Diagnostic Interview 3.0 on a random sample of 2804 individuals.

The study findings reported the lifetime prevalence of obsessive compulsive traits to be $13 \%$. The results showed that the greatest predictor factor was the presence of other mental disorders. Contrarily, Niller and Beck [28] which looked at a sample of seventy six college students who reported obsessive compulsive traits found that depression was not the main predictor, but the experience of guilt was, which relates to anxiety. In conjunction, confirming the findings of this study, some research has found a positive correlation between OCD and anxiety. Kessler, Berglund, Demler, Jin \& Walters [22] studied a sample of 9282 English speaking participants. They looked at the lifetime prevalence estimates of OCD in relation to other disorders. They found an estimated prevalence between OCD and anxiety disorders to be $28.8 \%$.

Furthermore, previous studies have confirmed a positive correlation between life event stress and OCD. Vidal-Ribas [29] looked at the impact of life events stress on lifetime prevalence and severity of obsessive-compulsive traits in a total of 22,084 MZ twins found that life events stress, especially that of abuse, family disruption and sexual abuse was significantly associated with the severity of obsessive-compulsive traits. Similarly, Maes, Mylle, Deilmeire and Altamura [21] studied the general population and found a significant correlation between life events, trauma and the development of OCD. It is therefore clear that the findings have confirmed the findings of previous research. However, contrary to previous research on clinical sample of OCD, there was no significant predictive effect between age, ethnicity, relationship status, educational level, employment status, family history of OCD, substance use and the development of obsessive-compulsive traits. This could be due to different predictors accounting for non-clinical OCD, possibly accounting for the transition phase between obsessive compulsive traits and clinical OCD.

To expand further, it can be suggested that this research study has expanded upon the limited research available regarding predictors of obsessive-compulsive traits as previous studies have mainly been carried out in patients with an OCD diagnosis. This study was conducted on university students with no known diagnosis of OCD as participants signed up on a voluntary basis. Moving onto the strengths of this study, the study was a quantitative study. By doing research quantitatively, a large sample 96 of participants was recruited. As a result, the findings are more likely to be valid and representative of the university student population. Additionally, strength is lies in the adaptation of multiple independent variables with one dependent variables. Consequently, wide range of possible predictor factors has been investigated allowing the use of multiple regression analysis to reveal the best predictor of the development of OCD.

Having said this, there are a number of limitations to this study. First of all, the study design was a cross-sectional. The disadvantage of using such design as suggested by Mann [30] is that it cannot be used to imply causation. Therefore, the results cannot be used to get to the root of obsessive compulsive traits and OCD. The results can only be used for information and as the basis for future research. Another limitation is that the sample only consisted of Kingston University psychology students, rather than the general university population. There is the possibility that psychology students alone are not representative of other students across the campus. Additionally, psychology students may be more likely to guess the aim of the study, possibly leading to the participants giving specific answers to the questionnaires due to social desirability bias.

In conjunction, the participants all received SONA credits as a reward for their participation. It could be suggested that the participants may have therefore been only motivated by the end reward of their participation and therefore put little to no thought into the responses they gave, leading to unreliable 
results. Furthermore, when it comes to the gender of the sample studied, there was an unequal ratio of females (76) and males (21). There were 76 females and 21 males. Consequently, the effects of gender as a predictor factor for the development of OCD was excluded from the main analysis which may act as a limitation as gender has previously been found to be a predictor of OCD as discussed in the introduction.

Additionally, it is challenging to identify whether the main predictor, depression predicts OCD or vice-versa. There is a possibility that depression develops following the obsessive compulsive traits or the diagnosis of OCD and therefore be a consequence or an effect of the disorder rather than a predictor.

Although this research study found a significant, positive correlation between life events stress and obsessive compulsive traits, the specific nature of the life events reported through self-report questionnaires was not investigated as only the total life events stress score was used for analysis. Therefore, the potential use of the data collected may be limited, especially if specific life events were to be a major contributor to the likelihood of an individual developing OCD. Lastly, when it came to statistical analysis, multiple regression, stepwise method was used which was used to report the strongest predictor of obsessive compulsive traits rather than a range of predictors as the aim of this study was to identify the strongest predictor of obsessive compulsive traits. This could act as a limitation and explain why previously found to be significant predictor factors including: age, ethnicity, relationship status, educational level, employment status, family history of OCD and substance use on the contrary was not significant predictors in this study.

Moving onto the possible implications of the study, the findings could be used to encourage healthcare professionals to screen for OCD in mental health checks and to recognize obsessive compulsive traits as a potential predictor for long term, diagnosable OCD, if left undiagnosed and untreated. Additionally, it could be suggested that individuals diagnosed with depression, should also be screened for OCD as based on the results, having depression may place an individual at a greater risk of developing OCD and act as a predictor. Lastly, the data could be used as evidence to promote greater mental health support to university students since OCD and obsessive compulsive traits were prevalent in $2.9 \%$. OCD currently affects $0.3-3 \%$ of the population. Therefore the $2.9 \%$ prevalence found within this sample puts the current sample on the far end of the prevalence risk on plectrums for OCD.

Moving onto direction for future research, a number of directions could take place. First of all, future research on the predictor factors of OCD should replicate the study on a larger cohort of students to further increase the validity and reliability of the validity. Additionally, future research should investigate into any predictor factors that may place students at a high risk of developing obsessive-compulsive traits and OCD as revealed by the results when placed on a risk spectrum. Another future consideration would be to consider studying specific subtypes of OCD such as contamination, hoarding, intrusive thoughts, checking, orderliness, symmetry and rumination to investigate whether predictor factors vary across them. Additionally, investigation into the influence of other possible predictor factors, such as birth socioeconomic status, birth order, family size, pregnancy and birth complications and specific at-risk genes should be studied.

When it comes to previous research on socioeconomic status, research has found major differences between individuals diagnosed with OCD and controls. Valleni-Basille et al. [20] studied adolescents from various socio-economic backgrounds and found that those from middle to high socio-economic class were at the highest risk of developing OCD. However, the sample size of this study was very small and therefore replication is necessary for conclusions to be drawn. Another predictor factor that should be studied in future research is birth order. Kayton and Bordge [31] found evidence to suggest that first-borns are at a greater risk of developing OCD. However, there is a lot of disagreement since more recent studies found no differences in birth order and the risk of developing OCD.

Additionally, research has shown that family size varies between individuals diagnosed with OCD and controls. Kayton and Borge [31] found that individuals diagnosed with OCD tends to come from smaller family. However, recent research has not shown this relationship. Therefore, by including birth order and family size in future research as possible predictors, it would help identify and confirm any differences that exist. Furthermore, when it comes to birth complications, studies have shown that it may act as potential predictor of the development of OCD. Geller, Klier and Neugebauer [32] studied a sample of miscarrying women and found that $3.5 \%$ of them experienced OCD recurrently when compared to $0.4 \%$ of the women in the community. Additionally, Vasconcelos et al. [33] looked at a sample of 68 patients diagnosed with OCD and 70 individuals that acted as controls and found that individual diagnosed with OCD had greater birth complications such as excessive weight, prolonger labour, premature birth and jaundice.

Lastly, another predictor factor that could be studied is specific at-risk genes as research has shown differences between individuals diagnosed with OCD and controls [3438]. A wide range of specific genes have been identified that requires replication and more research into these differences. To conclude, future direction of research should investigate into possible predictor factors accounting for the transition phase between the expression of obsessive compulsive traits in the general population and clinical OCD in clinical samples as this would allow healthcare professionals to tackle the signs early and begin treatment before the obsessive compulsive traits develop into diagnosable OCD. 


\section{Psychology and Behavioral Science International Journal}

\section{References}

1. Gottesman CG (1981) Twin and family studies of anxiety, phobic, and obsessive disorders. Anxiety-new research and changing concepts. Raven Press, New York, USA.

2. Pauls LD, Alsobrook PJ, Goodman W, Rasmussen S, Leckman FJ (1995) A family study of obsessive-compulsive disorder. Am J Psychiatry 152(1): 76-84.

3. Willour VL, Shugart YY, Samuels J, Grados M, Cullen B, et al. (2004) Replication Study Supports Evidence for Linkage to 9p24 in ObsessiveCompulsive Disorder. Am J Hum Genet 75(3): 508-513.

4. Delorme R, Betancur C, Wagner M, Krebs MO, Gorwood P, et al. (2005) Support for the association between the rare functional variant I425V of the serotonin transporter gene and susceptibility to obsessive compulsive disorder. Mol Psychiatry 10(12): 1059-1061.

5. Ozaki N, Goldman K, Kaye WH, Plotnicov K, Greeberg DB, et al. (2003) Serotonin transporter missense mutation associated with a complex neuropsychiatric phenotype. Mol Psychiatry 8(11): 933-936.

6. Arnold P, Rosenberg D, Mundo E, Tharmalingam S, Kennedy J, et al (2004) Association of a glutamate (NMDA) subunit receptor gene (GRIN2B) with obsessivecompulsive disorder: a preliminary study Psychopharmacology 174(4): 530-538.

7. Marazziti D, Hollander E, Lensi P, Ravagli S, Cassano GB (1992) Peripheral markers of serotonin and dopamine function in obsessivecompulsive disorder. Psychiatry Res42(1): 41-51.

8. Mundo E, Bellodi L, Smeraldi E (1995) Effects of acute intravenous clomipramine on obsessive-compulsive symptoms and response to chronic treatment. Biol Psychiatry 38(8): 525-531.

9. David KL, Charney D, Coyle JT, Nemeroff C (2002) The Pathophysiology and Genetics of OCD. Neuropsychopharmacology: The fifth generation of progress. American College of Neuropsychopharmacology. Lippincott/Williams \& Wilkins, Philadelphia, USA.

10. Saxena S, Rauch SL (2000) Functional Neuroimaging and the Neuroanatomy of Obsessive-Compulsive Disorder. Psychiatr Clin North Am 23(3): 563-586.

11. Simon D, Adler N, Kaufmann C, Kathmann N (2014) Amygdala hyperactivation during symptom provocation in obsessive-compulsive disorder and its modulation by distraction. Neuroimage Clin 4: 549557

12. Heyman I, Fombonne E, Simmons H, Ford T, Meltzer H, et al. (2001) Prevalence of obsessive-compulsive disorder in the British nationwide survey of child mental health. Br J Psychiatry 179(4): 324-329.

13. Williams TM, Turkheimer E, Schmidt MK, Oltmanns FT (2005) Ethnic Identification Biases Responses to the Padua Inventory for ObsessiveCompulsive Disorder. Assessment 12(2): 174-185.

14. Cilliçilli AS, Telcioglu M, Așkin R, Kaya N, Bodur S, et al. (2004) Twelvemonth prevalence of obsessive-compulsive disorder in Konya, Turkey. Compr Psychiatry 45(5): 367-374.

15. Crino R, Slade T, Andrews G (2005) The Changing Prevalence and Severity of Obsessive-Compulsive Disorder Criteria from DSM-III to DSM-IV. Am J Psychiatry 162(5): 876-882.

16. Fontenelle LF, Mendlowicz MV, Marques C, Versiani M (2004) Transcultural aspects of obsessive-compulsive disorder: a description of a Brazilian sample and a systematic review of international clinical studies. J Psychiatr Res 38(4): 403-411.

17. Degonda M, Wyss M, Angst J (2003) The Zurich Study XVIII Obsessivecompulsive disorders and syndromes in the general population. European Archives of Psychiatry and Clinical Neuroscience 243(1): 16-22.
18. Douglass HM, Moffitt TE, Dar R, McGee R, Silva P (1995) ObsessiveCompulsive Disorder in a Birth Cohort of 18-Year-Olds: Prevalence and Predictors. J Am Acad Child Adolesc Psychiatry 34(11): 1424-1431.

19. Moylan S, Jacka FN, Pasco JA, Berk M (2013) How cigarette smoking may increase the risk of anxiety symptoms and anxiety disorders: a critical review of biological pathways. Brain Behav 3(3): 302-326.

20. Valleni-Basile LA, Garrison CZ, Waller JL, Addy CL, McKeown RE, et al. (1996) Incidence of Obsessive-Compulsive Disorder in a Community Sample of Young Adolescents. J Am Acad Child Adolesc Psychiatry 35(7): 898-906

21. Maes M, Mylle J, Delmeire L, Altamura C (2000) Psychiatric morbidity and comorbidity following accidental man-made traumatic events: incidence and risk factors. Eur Arch Psychiatry Clin Neurosci 250(3): 156-162.

22. Kessler RC, Berglund P, Demler O, Jin R, Walters EE (2005) Lifetime Prevalence and Age-of-Onset Distributions of DSM-IV Disorders in the National Comorbidity Survey Replication. Arch Gen Psychiatry 62(6): 593-602.

23. Kroenke K, Spitzer RL (2002) The PHQ-9: A New Depression Diagnostic and Severity Measure. Psychiatric Annals 32(9): 509-515.

24. Spitzer RL, Kroenke K, Williams JB, Löwe B (2006) A Brief Measure for Assessing Generalized Anxiety Disorder. Arch Intern Med 166(10): 1092-1097.

25. Foa EB, Kozak MJ, Salkovskis PM, Coles ME, Amir N (1998) The validation of a new obsessive-compulsive disorder scale: The Obsessive-Compulsive Inventory. Psychological Assessment 10(3): 206-214.

26. Holmes TH, Rahe RH (1967) The social readjustment rating scale. J Psychosom Res 11(2): 213-218.

27. Fullana M, Vilagut G, Rojas-Farreras S, Mataix-Cols D, De Graaf R, et al. (2010) Obsessive-compulsive symptom dimensions in the general population: Results from an epidemiological study in six European countries. J Affect Disord 124(3): 291-299.

28. Niler ER, Beck SJ (1989) The relationship among guilt, dysphoria, anxiety and obsessions in a normal population. Behav Res Ther 27(3): 213-220.

29. Vidal-Ribas P, Stringaris A, Rück C, Serlachius E, Lichtenstein P, et al. (2015) Are stressful life events causally related to the severity of obsessive-compulsive symptoms? A monozygotic twin difference study. Eur Psychiatry 30(2): 309-316.

30. Mathews CA, Jang KL, Hami S, Stein MB (2004) The structure of obsessionality among young adults. Depress Anxiety 20(2): 77-85.

31. Kayton L, Borge GF (1967) Birth Order and the Obsessive-Compulsive Character. Arch Gen Psychiatry 17(6): 751-754.

32. Geller AP, Klier MC, Neugebauer R (2001) Anxiety Disorders Following Miscarriage. J Clin Psychiatry 62(6): 432-438.

33. Vasconcelos MS, Sampaio AS, Hounie AG, Akkerman F, Curi M, et al. (2006) Prenatal, Perinatal, and Postnatal Risk Factors in ObsessiveCompulsive Disorder. Biol Psychiatry 61(3): 301-307.

34. American Psychiatric Association (2013) Diagnostic and statistical manual of mental disorders: DSM-5. American Psychiatric Association, Washington, USA.

35. Fontenelle LF, Hasler G (2008) The analytical epidemiology of obsessive-compulsive disorder: Risk factors and correlates. Prog Neuropsychopharmacol Biol Psychiatry 32(1): 1-15.

36. Nestadt G, Grados M, Samuels JF (2010) Genetics of ObsessiveCompulsive Disorder. Psychiatr Clin North Am 33(1): 141-158. 
37. Pooley EC, Fineberg N, Harrison PJ (2007) The met158 allele of catechol-Omethyltransferase (COMT) is associated with obsessivecompulsive disorder in men: case- control study and meta-analysis. Mol Psychiatry 12(6): 556-561.
38. Samuels JF, Riddle MA, Greenberg BD, Fyer AJ, McCracken JT, et al (2006) The OCD collaborative genetics study: Methods and sample description. Am J Med Genet B Neuropsychiatr Genet 141B(3): 201207.

\begin{tabular}{l} 
Your next submission with Juniper Publishers \\
will reach you the below assets \\
- Quality Editorial service \\
- Swift Peer Review \\
- Reprints availability \\
- E-prints Service \\
- Manuscript Podcast for convenient understanding \\
- Global attainment for your research \\
- Manuscript accessibility in different formats \\
( Pdf, E-pub, Full Text, Audio) \\
- Unceasing customer service \\
Track the below URL for one-step submission \\
https://juniperpublishers.com/online-submission.php \\
\hline
\end{tabular}

